\title{
Understanding hearing impairment through model predictions of brainstem
}

responses

Sarah Verhulst and Hari BharadwajGolbarg MehraeiBarbara Shinn-Cunningham

Citation: Proc. Mtgs. Acoust. 19, 050182 (2013); doi: 10.1121/1.4800566

View online: http://dx.doi.org/10.1121/1.4800566

View Table of Contents: http://asa.scitation.org/toc/pma/19/1

Published by the Acoustical Society of America 


\section{Proceedings of Meetings on Acoustics}
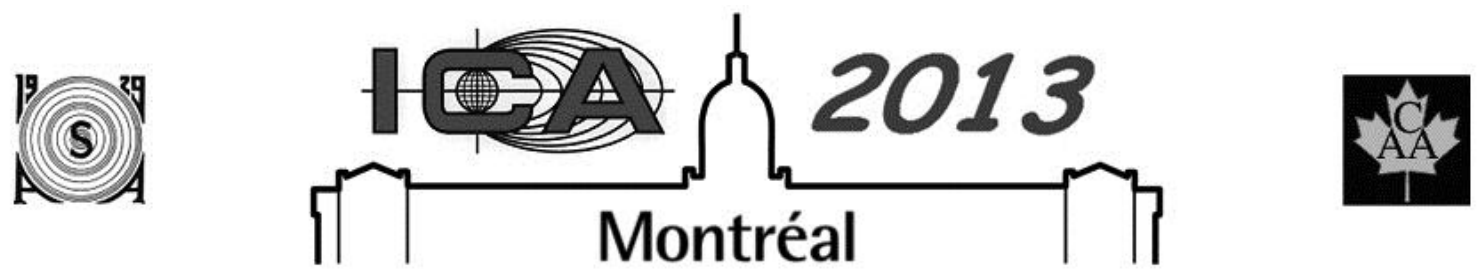

ICA 2013 Montreal

Montreal, Canada

2 - 7 June 2013

Psychological and Physiological Acoustics

Session 4pPP: Computational Modeling of Sensorineural Hearing Loss: Models and Applications

\section{4pPP7. Understanding hearing impairment through model predictions of brainstem} responses

Sarah Verhulst*, Hari Bharadwaj, Golbarg Mehraei and Barbara Shinn-Cunningham

*Corresponding author's address: Center for Computational Neuroscience and Neural Technology, Boston University, 677 Beacon St, Boston, MA 02215, save@bu.edu

Latencies of auditory brainstem response (ABR) wave-V decrease with increasing stimulus level, an effect often ascribed to broadened auditory filters. Following this hypothesis, hearing- impaired subjects with broad auditory filters should exhibit shorter wave-V latencies than normal-hearing listeners. Hearing anomalies resulting from the preferential degradation of low spontaneous rate (LS) auditory nerve (AN) fibers with intact thresholds have recently received attention. However, their effect on the ABR wave-V latency are yet to be elucidated. Here, a model of ABR investigates the relationships between wave-V latency and various forms of hearing damage. ABR wave-Vs are predicted from a model consisting of a nonlinear cochlear model (Verhulst et al., 2012, JASA 132), an IHC/AN synapse model (Heinz et al., 2001, ARLO 5), and a model of the cochlear nucleus (CN) and inferior colliculus (IC) (Nelson and Carney, 2004, JASA 116). Simulations show that on a single-unit level, latency-with-level functions of the AN and IC reflect the width of the underlying auditory filter, an effect that is greatly reduced in simulated ABR wave-V latencies. The adopted modeling approach can improve our understanding of ABR wave-V latency and thereby enhance its predictive power in the diagnostics of various forms of hearing impairment.

Published by the Acoustical Society of America through the American Institute of Physics 


\section{INTRODUCTION}

Latencies of auditory brainstem response (ABR) wave- $\mathrm{V}$ decrease by $1.5-2 \mathrm{~ms}$ for a stimulus level increase of $40 \mathrm{~dB}$ (Dau, 2003; Elberling et al., 2010). Based on the correspondence between ABR wave-V latency and that of tone-burst-evoked otoacoustic emissions (Neely et al., 1988; Harte et al. 2009), the wave-V latency decrease with level has often been ascribed to broadened auditory filters. Following this hypothesis, hearing-impaired subjects with broad auditory filters should exhibit shorter wave-V latencies than normal-hearing listeners. Unfortunately, experimental studies fail to demonstrate a clear relation between decreased ABR latencies and widened auditory filters as a consequence of sensorineural hearing loss. For instance, Don et al. (1998) showed derived-band ABR wave- $\mathrm{V}$ latencies to $93 \mathrm{~dB}$ peSPL clicks that decreased with increasing pure-tone thresholds for high-pass noise cutoff frequencies of 0.7 and $1.4 \mathrm{kHz}$, in support of the hypothesis. Yet, Donaldson and Ruth (1996) did not observe an effect of hearing loss on the derived-band ABR wave-V latency. When plotted as a function of dB SPL, only a small shift $(0.2 \mathrm{~ms})$ towards shorter latencies in the ABR wave-I latency-with-level curve was observed in another study of chinchillas with noise-induced hearing loss (Henri et al., 2011). In agreement with these findings, similar latencywith-level slopes for human ABR wave- $\mathrm{V}$ were found for a normal-hearing group and a group with a flat sensorineural hearing loss (Serpanos et al., 1997). However, in contrast to linear filter theory, the hearing-impaired and normal-hearing groups showed similar latency values. Even stranger, the group of listeners with sloping audiograms showed a larger spread in wave- $\mathrm{V}$ latency values that often exceeded the latencies obtained for normal hearing listeners when plotting the results as a function of $\mathrm{dB}$ HL (hearing level). A recent study confirms this observation, showing that compared to normal-hearing listeners, hearing-impaired listeners have greater ABR wave$\mathrm{V}$ latencies $(0.5$ to $1 \mathrm{~ms})$ for moderate stimulation levels of $60-70 \mathrm{~dB}$ peSPL, while showing similar or shorter latencies for the 80-90 dB peSPL levels (Strelcyk et al., 2009).

The above experimental studies suggest that the relationship between auditory filter width and the latency of ABR wave- $\mathrm{V}$ for sensorineural hearing loss is perhaps not as straightforward as predicted by linear filter theory. This departure from theoretical predictions may reflect recent results showing that noise exposure leads to a preferential loss of low spontaneous-rate (SR) auditory-nerve (AN) fibers over high SR fibers (Furman, 2013), even before permanent threshold shifts manifest (Kujawa and Liberman, 2009). As the latency of firing of LS fibers is generally delayed to that of HS fibers in the auditory nerve (Rhode and Smith, 1985), the loss of specific populations of fibers may affect the latency of the ABR. As LS fibers have higher thresholds than HS fibers (Liberman, 1978), their loss and corresponding decrease in overall firing latency as the HS fibers take over is probably more pronounced for moderate to high stimulus levels.

These results suggest that hearing loss in humans may not only cause wider auditory filters due to changes in cochlear amplification, but may also occur because of a loss of LS auditory nerve fibers. These different mechanisms of hearing loss may result in different changes in ABR measures. ABR could thus prove useful, clinically, in diagnosing different forms of hearing loss, including losses that do not currently show up in ordinary hearing screenings. This study investigates ABR wave-V latency under normal and impaired conditions in a model that incorporates populations of both HS and LS fibers. The model, which combines existing models of the cochlea (Verhulst et al., 2012), auditory nerve and synapse (Heinz et al. 2001), ventral cochlear nucleus and inferior colliculus (Nelson and Carney, 2004) was able to account for a 2-ms decrease in ABR wave-V latency for stimulus levels between 60 and $100 \mathrm{~dB}$ peSPL for normal-hearing ears. This model thus provides an improvement over existing ABR models that can only account for a latency decrease of $0.22 \mathrm{~ms}$ (Dau, 2003) or $0.6 \mathrm{~ms}$ (Rønne et al., 2012). We also investigated how ABR wave-V latency was altered as a consequence of widened auditory filters, the loss of LS fibers, and the presence of background noise. Single-unit responses at the level of the basilar membrane (BM), auditory nerve (AN), and inferior colliculus (IC) are simulated, and their latencies compared to that of the summed responses at the level of the AN and IC (i.e., ABR wave-V).

\section{METHODS}

The existing ABR models of Dau (2003) and Rønne et al. (2012) were extended to account for a 2-ms latency decrease for a $40-\mathrm{dB}$ click-level increase for normal hearing listeners in the simulated ABR wave-V. These alterations are summarized in the model schematic represented in Fig.1(a) and included: (i) The adoption of a transmission-line model of the cochlea (Verhulst et al., 2012) to replace the parallel auditory filter bank that was 
used in the models of Heinz et al. (2001), Dau (2003), Zilany et al., (2009) and Rønne et al. (2012). (ii) The choice of using the inner-hair cell (IHC) and auditory-nerve synapse model by Heinz et al. (2001), over that of Zilany et al. (2009). And (iii), the use of the functional ventral cochlear nucleus (VCN) and inferior colliculus (IC) model to represent the ABR wave III and V, respectively, over the unitary response approach employed by Dau et al. (2003) and Rønne et al. (2012).
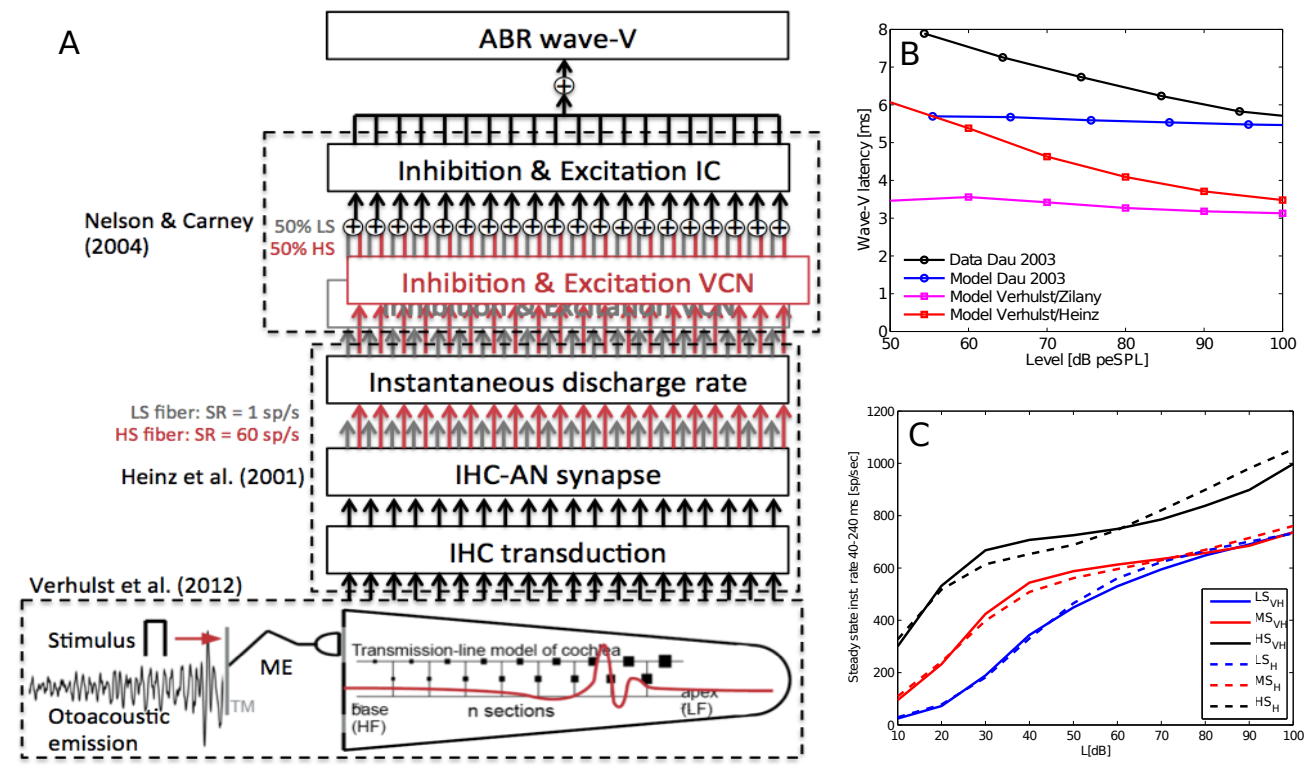

FIGURE 1. (a) Schematic of the ABR wave-V model. (b) Comparison between the ABR wave-V latency data of Dau (2003) and simulated wave-V ABR latency in three model implementations: the Dau (2003) ABR model, the model presented in (a), and the model in schematic (a) using the Zilany et al. (2009) instead of the Heinz et al. (2001) implementation for the IHC/AN. (c) Ratelevel functions for the instantaneous auditory-nerve firing rate in the model of Heinz et al. (2001, dashed) compared to those obtained with the model in schematic A (solid), for auditory nerve fibers with three spontaneous rates: LS $1 \mathrm{sp} / \mathrm{s}$, MS 5sp/s and HS $60 \mathrm{sp} / \mathrm{s}$. The steady state rate was determined from the 40 to $240 \mathrm{~ms}$ window of the instantaneous firing rate to continued sinusoidal stimulation.

\section{Basilar-membrane processing}

The cochlear stage of the ABR models of Dau (2003) and Rønne et al. (2012) were based on the implementations of Zhang et al. (2001) and Ibrahim and Bruce (2010), respectively. These models represent basilar-membrane processing as a set of parallel auditory filters that consist of a signal and control path representing the auditory filter (gammatone or gammachirp), BM compression, and the effects of basilar-membrane coupling. Even though these models were adjusted to reflect human auditory filter tuning (Q) values, the large number of free parameters in the signal and control path make it difficult to obtain a broadband cochlear response that complies with noninvasive measurements human cochlear travel time, compression and two-tone suppression. This approach can be improved when using a transmission-line model of the cochlea that, (i) because of its serial implementation accounts for frequency glides, two-tone suppression, and distortion products without the need of a control path when representing BM processing as a cascade of nonlinearly growing band-pass filters, and (ii), can be adjusted to simulate otoacoustic emissions such that human otoacoustic emission data can be used to calibrate the models tuning, compression, and travel time characteristics (e.g. van Hengel, 1996; Moleti et al., 2009; Epp et al., 2009; Verhulst et al., 2012). As this study predominantly uses broadband stimuli such as clicks and noise, the model of Verhulst et al. (2012) was adopted because its nonlinear characteristics were designed to reflect BM impulse response characteristics, and it was calibrated using human click-evoked otoacoustic emission data. 80- $\mu$ s condensation clicks were presented to the input of the middle-ear stage of the model as a pressure signal corresponding to dB peSPL levels. BM displacement patterns were obtained for 1000 cochlear sections with CFs between $140 \mathrm{~Hz}$ and $20.7 \mathrm{kHz}$, and the dominant double pole $\alpha^{*}$ of the BM admittance was set to 0.06 at low stimulus levels, leading to auditory filters with a $\mathrm{Q}_{\mathrm{ERB}}$ of 10 across the cochlear partition. $\mathrm{Q}_{\mathrm{ERB}}$ decreased with increasing stimulus level for stimulus levels between 30 and $97 \mathrm{~dB}$ SPL, and a BM compression slope of $0.4 \mathrm{~dB} / \mathrm{dB}$ was applied. 


\section{IHC and AN model}

BM displacement patterns of 500 cochlear sections, logarithmically spaced with characteristic frequencies (CF) between $140 \mathrm{~Hz}$ and $20.7 \mathrm{kHz}$, were first scaled to match the pressure signal at the output of the signal path filter (and before application of the IHC stage) in Heinz et al. (2001). As the auditory filter bank in Heinz et al. (2001) works with pressure signals and the Verhulst et al. (2012) model simulates BM displacement patterns, the models were matched at the input of the IHC such that the outputs of the IHC stage in the models were identical for a 10-dB SPL pure tone with center frequency of $1 \mathrm{kHz}$. The resulting scaling factor $\left(2.0688 \mathrm{e}^{5}\right)$ was applied to all CFs and BM displacements before they were fed to an asymmetric logarithmic compressive function (Zhang et al., 2001) and low-pass filter with cut-off frequency of $4.5 \mathrm{kHz}$ (Heinz et al., 2001) to simulate IHC processing in the model.

Instantaneous discharge rates in the auditory nerve were simulated using a time-varying three-store diffusion model (Westerman and Smith (1988)) that has been used in several studies to simulate the IHC/AN synapse (Heinz et al. (2001); Zhang et al., (2001); Zilany et al., (2009)). The parameters were chosen to be identical to those in a past study that achieved realistic steady-state rate-level functions for pure-tone stimulation (Heinz et al., 2001). Fig.1(c) shows rate-level curves for three auditory nerve fibers with low $(1 \mathrm{sp} / \mathrm{s})$, medium $(5 \mathrm{sp} / \mathrm{s})$ and high $(60 \mathrm{sp} / \mathrm{s})$ spontaneous rates. Firing rates of the LS fiber increased with a shallower slope than that of the HS fiber, and saturated at $75 \%$ of the rates of the HS fiber, in qualitative agreement with AN saturation rates found in Liberman (1978) and Rhode and Smith (1985). Shallower rate-level slopes were found experimentally in Sachs and Abbas (1974) for high versus low threshold AN fibers, and for LS fibers that generally had higher thresholds than HS AS fibers (Liberman, 1978). The current model implementation did not account for refractoriness and thereby does not generate AN spike histograms, nor did it include power-law adaptation (e.g., as described in Zilany et al., 2009).

\section{CN and IC model}

Instantaneous $\mathrm{AN}$ firing patterns were used as input to a functional model of the ventral cochlear nucleus (VCN) and inferior colliculus (IC; Nelson and Carney, 2004). For each of the $500 \mathrm{CFs}$ simulated with the IHC/AN model, discharge patterns for two spontaneous rates were obtained: LS (1 sp/s) and HS (60 sp/s). These two different discharge patterns were each fed to both an excitatory ( $\tau$ of $0.5 \mathrm{~ms}$ ) and an inhibitory ( $\tau$ of $2 \mathrm{~ms}$ ) input of the CN model. For each CF, the two CN outputs for each AN fiber type (LS and HS) were averaged before being fed to the IC stage of the model. The IC stage of the model consists of an excitatory and inhibitory pathway similar to that of the $\mathrm{CN}$, with the difference that the strength of the inhibitory pathway was stronger than the excitatory one in the IC, whereas in the $\mathrm{CN}$, the opposite is true. The main difference between representing the VCN and IC stage with this functional model (Nelson and Carney, 2004) rather than using a unitary response model (e.g., Dau, 2003; Rønne et al., 2012) is that the current approach makes no assumptions about the shape of the grand average ABR waveform from which the unitary response is derived. However, a drawback of the CN/IC approach is that the wave-III (CN) and wave-V (IC) amplitude and latency are obtained separately. A comparison of wave-V latency for the current model and one of the unitary models (Dau, 2003) is shown in Fig.1(b). Whereas the unitary model was unable to account for a 2-ms decrease in wave- $\mathrm{V}$ latency for a $40-\mathrm{dB}$ increase in stimulus level, the current model driving the $\mathrm{CN} / \mathrm{IC}$ model obtained such a latency shift. Note that a more recent IHC/AN implementation (Zilany et al., 2009) was not used in the current study because it was unable to account for the latency shift when using the instantaneous discharge rate as an input to the CN/IC model (see Fig.1(b) Verhulst/Zilany).

\section{Hearing Impairment}

Hearing impairment was modeled with two independent contributing factors: (i) a loss of cochlear gain that leads to widened auditory filters and increased detection thresholds, and (ii) the preferential loss of LS fibers following noise exposure. To simulate the loss of cochlear gain, the dominant double pole $\alpha^{*}$ of the BM admittance was changed from a value of 0.06 to a value of 0.1 , leading to auditory filters with a $\mathrm{Q}_{\text {ERB }}$ of 5.5 (Verhulst et al., 2012). As changing $\alpha^{*}$ affects both the stiffness and damping of the BM admittance, widened auditory filters are accompanied by overall lower BM displacement levels than would be obtained for filters with higher QERB values. Consequently, higher stimulus levels are needed to achieve the same output level at the IHC stage of the model, thereby simulating elevated detection thresholds. As the model employs constant $Q$ values across the cochlear partition, a flat-spectrum hearing loss was simulated. To investigate the loss of specific auditory nerve populations, all model simulations were performed for three model implementations. In the first, the total composition of fibers 
was comprised of $50 \%$ of HS fibers and $50 \%$ LS fibers; in the other two implementations, the AN fibers were either $100 \%$ LS fibers or $100 \%$ HS fibers.

\section{RESULTS}

\section{Single-unit Responses}

Single-unit responses were simulated at four levels of processing (BM, AN, CN and IC) for a characteristic place of $1 \mathrm{kHz}$. Results were normalized to the local maximum of the waveform for different stimulus levels. Fig.2(a) shows single-unit responses to clicks of varying intensity for a model of a normal-hearing ear. As stimulus level increases, BM impulse responses become shorter and peakier, in agreement with past experimental observations (Recio and Rhode, 2000). For low stimulus levels, the LS and HS AN show maximal excitation near the onset of the $\mathrm{BM}$ waveform, likely because the fibers are close to firing at their spontaneous rate. For medium stimulus levels (40 and $60 \mathrm{~dB}$ ), the LS fibers tend to fire near the maximum of the BM waveform, whereas the HS fibers fire at the first peak of the waveform. The longer firing latencies of the LS fibers versus the HS fibers agree with past physiological data (Rhode and Smith, 1985). For high stimulus levels, both the LS and HS AN fibers show a maximal firing rate at the first peak of the BM waveform. The latencies of the maxima of the $\mathrm{CN}$ and IC waveform generally follow the maxima of the AN waveform by a fixed delay, except for the lowest stimulation levels, at which the CN and IC maxima occur earlier because the AN firing patterns are small and close to spontaneous rate. Hearing impairment (Fig.2(b)) primarily affects the duration and shape of the BM impulse responses. As a result, waveforms are peakier than those obtained for the normal-hearing model (Fig.2(a)), and the AN fibers discharge maximally near the onset peak of the BM displacement pattern, regardless of fiber type.

In the presence of ongoing background noise (that started $50 \mathrm{~ms}$ before the onset of the click), the different AN fiber types behave differently in response to a fixed level click (90 dB peSPL). For a HS AN fiber, the click no longer causes a distinguishable change in the response compared to the background noise alone at noise levels of 76 $\mathrm{dB}$ SPL or greater. However, for the LS fiber, the click response is more robust at high noise levels. This effect may arise because the background noise causes the HS AN fiber to saturate for lower stimulus levels compared to the LS fiber (see rate-level curves of Fig.1(c)). Fig.3 summarizes the peak latencies for a single-unit BM, AN, and IC responses at $1 \mathrm{kHz}$ for the waveforms visualized in Fig.2.
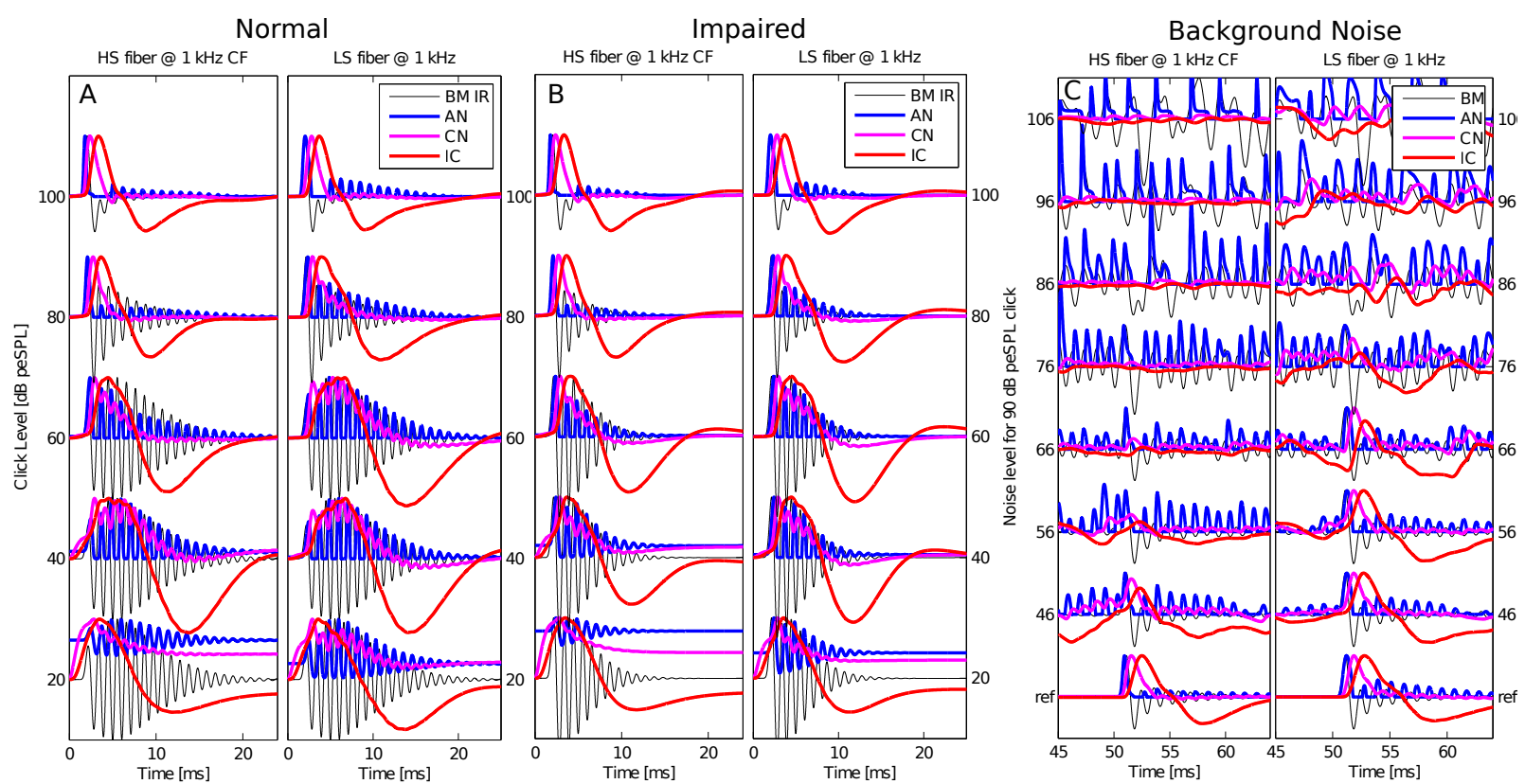

FIGURE 2. Simulations of single-unit responses at the level of the basilar membrane (black), auditory nerve fiber (blue), cochlear nucleus (magenta) and inferior colliculus (red) for two types of auditory nerve fibers: LS 1sp/s and HS 60sp/s. (a) responses to a $80-\mu$ s-click of various intensity in a normal hearing model that has sharply tuned auditory filters $\left(Q_{E R B}\right.$ of 10$)$. (b) responses to the same clicks in an impaired model that has decreased thresholds and widened auditory filters ( $\mathrm{Q}_{\mathrm{ERB}}$ of 5.5). (c) responses to a 90-dB-peSPL click embedded in white noise of various intensity. 

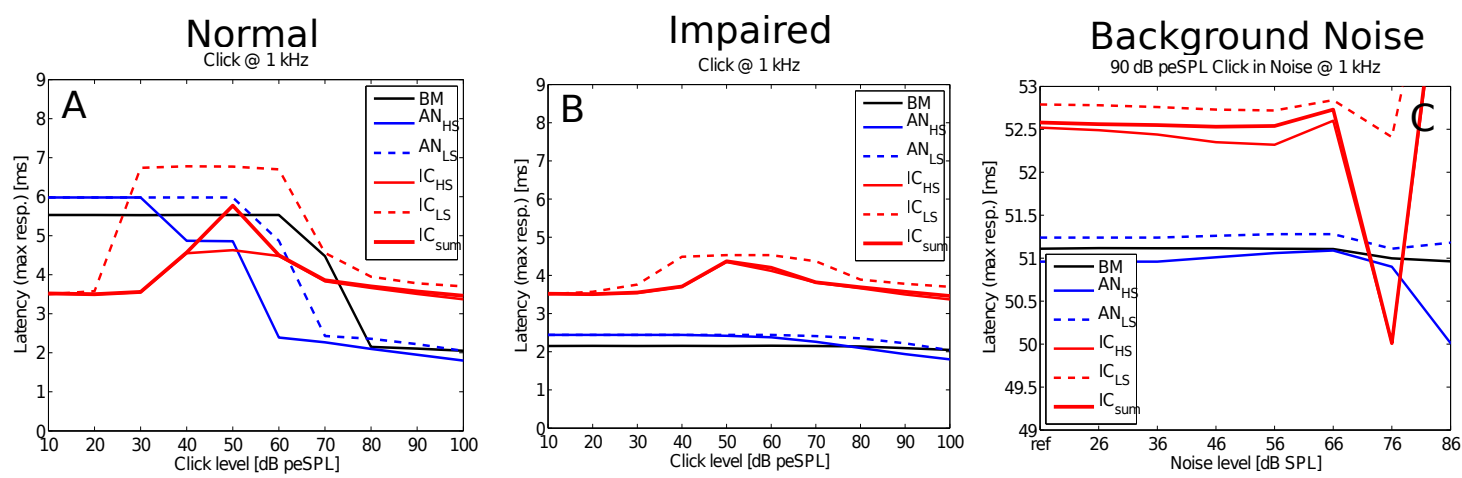

FIGURE 3. Peak latency for the single-unit response analyzed at three levels of processing: basilar membrane (black), auditory nerve (blue), and inferior colliculus (red). Subscripts LS and HS reflect models that contain either low (1 sp/s) or high (60 sp/s) spontaneous rate fibers. The $\mathrm{IC}_{\text {sum }}$ response was calculated for a model driven by $50 \%$ LS fibers and $50 \%$ HS fibers. (a) Responses to a 80 - $\mu$ s-click of various intensity in a normal-hearing ear model that has sharply tuned auditory filters $\left(Q_{\text {ERB }}\right.$ of 10$)$. (b) Responses to the same clicks as in a), but for an impaired model with elevated detection thresholds and wider auditory filters $\left(\mathrm{Q}_{\mathrm{ERB}}\right.$ of 5.5) than the normal-ear model. (c) Responses to a $90 \mathrm{~dB}$ peSPL click embedded in white noise of various intensities.

At the single-unit level, the AN and IC latency-with-level function follows the BM impulse response waveform shape. For the normal hearing model, the peak of the BM impulse response decreased from 5.5 to $2 \mathrm{~ms}$ as the stimulus level increased from $60 \mathrm{~dB}$ to greater levels; this was accompanied by a latency decrease of 4 and $3 \mathrm{~ms}$ for the LS and HS AN fiber responses, respectively. For the hearing-impaired model, the BM impulse response peak is nearly constant with level because of the overall wider auditory filters, which translates into a latency decrease of less than $0.5 \mathrm{~ms}$ in the AN latency. The IC latencies basically follow the BM and AN latencies with a constant offset, except for stimulus levels below $40 \mathrm{~dB}$, where they are driven by the spontaneous discharge of the AN fibers (see Fig.2(a) and (b)). LS fibers generally fired with longer latencies than HS fibers; however, latency-with-level functions were similarly shaped for the two populations of fibers. The IC latencies of the model with $50 \%$ LS and $50 \%$ HS fibers (i.e., $\mathrm{IC}_{\text {sum }}$ ) followed the behavior of the $100 \% \mathrm{HS}$ model except for a stimulus level of $50 \mathrm{~dB}$ peSPL. This finding suggests that a loss of LS fibers at a specific CF does not affect the IC latency-with-level function much at that single-unit level.

The single-unit responses to clicks presented in background noise (Fig.2 (c)) did not show large changes in either BM, AN, or IC latency as background noise levels increased. For noise levels above $66 \mathrm{~dB}$ SPL, the clicks did generally not cause a noticeable change in responses relative to the response to the background noise alone; which resulted in latency values that did not change systematically with noise level. The increase in response latency recorded from an IC chopper neuron for clicks embedded in increasing noise levels (Burkard and Palmer, 1997) was not observed in the simulations presented here.

\section{Wide-band Responses}

Even though the latencies of single-unit responses for both the normal-hearing and hearing-impaired models followed the BM impulse response maxima, and hence reflect the width of the underlying auditory filters, it is not clear that the ABR wave-V will reflect this dependence. The simulated ABR wave-V latencies are found by summing all simulated single-unit responses at the level of the IC, and may thus average out many of the dependencies that are observed in single units. Simulated ABR wave-V latencies are shown for the normal-hearing (NH) and hearing-impaired (HI) models in Fig.4(a).

The simulated ABR wave- $\mathrm{V}$ response shows a $\sim 2$-ms decrease in latency with increasing stimulus level, in agreement with experimental ABR wave-V latency data (Dau, 2003; Strelcyk et al., 2009). Hearing impairment decreases the ABR wave- $V$ response by $0.2 \mathrm{~ms}$ for a stimulus level of $50 \mathrm{~dB}$ peSPL after which the difference in latency between the hearing-impaired and normal-hearing model gradually disappears. This lack of dependence on stimulus level agrees with data showing that at high stimulus levels, the wave-V latencies are similar for NH and HI 

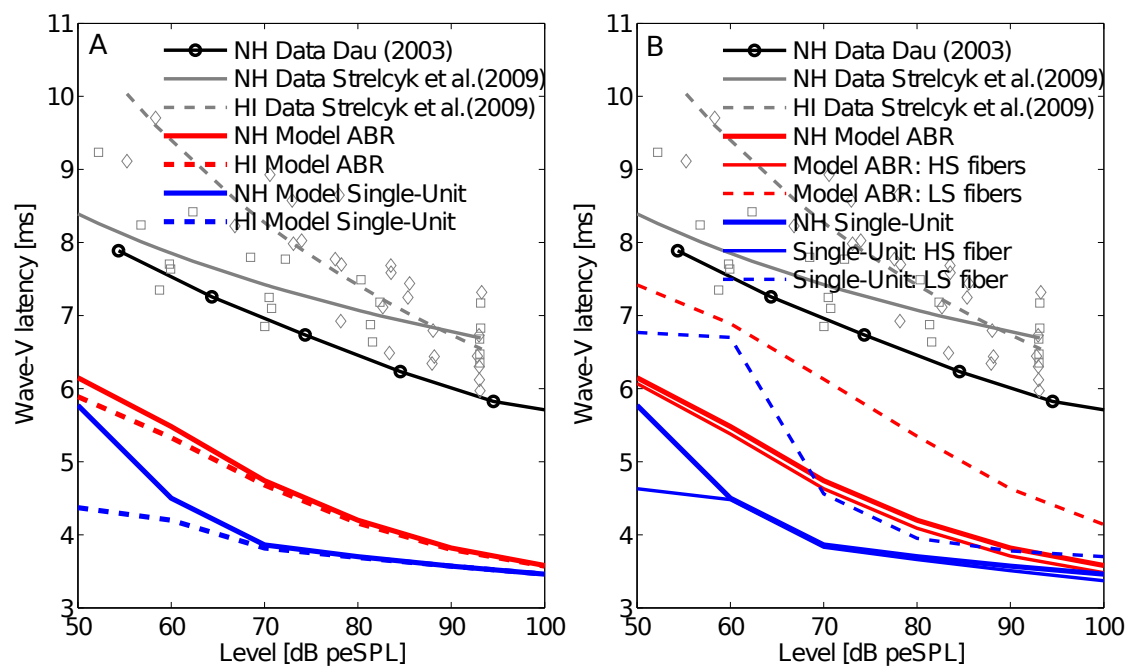

FIGURE 4. (a) Comparison between simulated ABR wave-V data to clicks for a normal-hearing and impaired model with the experimental studies by Dau (2003) and Strelcyk et al. (2009). A power-law function was fit to the group data for the normalhearing and impaired subjects of Strelcyk et al. (2009) (gray lines). Both the latencies for the wide-band response (i.e., ABRwave $\mathrm{V}$ in red) and the single-unit response ( $1 \mathrm{kHz} \mathrm{CF}$ in blue) are shown. (b) Influence of the population of LS and HS fibers on the latency of the wide-band (ABR) and single-unit responses (at $1 \mathrm{kHz}$ ) for an otherwise normal-hearing model.

listeners (Strelcyk et al., 2009). At high stimulus levels, the auditory filters for NH and HI listeners are wide and characterized by peaky impulse responses (see Fig.2(a) and (b)); therefore, the latency of firing in the AN is not expected to change significantly with level (see also Fig.3(a) and (b)). As observed from the single-unit responses in Fig.3(a) and (b) (plotted again in Fig.4(a) for the $1 \mathrm{kHz}$ location), latencies shift with level in the mid-range of stimulus intensities, an effect that can be attributed to widening of the auditory filters in the hearing-impaired simulation. The simulated ABR wave-V shows an overall latency decrease of only $0.2 \mathrm{~ms}$ at these stimulation levels. It is possible that the summation of synchronous energy across the simulated CF channels is responsible for this smaller latency difference than observed for the 1-kHz single-unit response. Specifically, effects may differ with position along the basilar membrane, so that the summation across peripheral channels washes out stimulus level effects. Indeed, cochlear dispersion will result in the high-frequency channels firing more synchronously across a section of the cochlea in response to click stimuli than will low frequency channels (e.g. Dolan et al., 1983).

Because dispersion is likely to reduce the contribution of low-frequency channels to the synchronous responses that dominate the ABR wave-V, the summed IC response will be dominated by information in high-frequency channels. These high-frequency channels generally have shorter-duration impulse responses than low-frequency channels. Wider auditory filters would not result in large latency shifts for these frequencies, possibly explaining the difference between the single-unit and wide-band responses. At moderate stimulus levels, the ABR wave-V latency does not follow the trend of increased latencies for the hearing-impaired listeners (e.g., see Strelcyk et al., 2009). Possible reasons for this discrepancy are: (i) they looked at the derived-band ABR latency in the presence of a highpass noise with a cut-off frequency of $2.8 \mathrm{kHz}$, rather than at the broadband ABR waveform, and (ii) the hearing losses they considered were sloping high-frequency losses, rather than the flat losses examined in this study.

Effects of different populations of AN fibers were investigated in Fig.4 (b). The ABR wave-V latency of the model with $50 \%$ HS and $50 \%$ LS fibers is similar to the behavior of the model with $100 \%$ HS fibers. The contribution of the LS fibers to the total model response was larger for the single-unit response in Fig.4(b) than for the wide-band IC response. The loss of the whole population of LS fibers in the model was shown to decrease the overall wave-V latency by only $0.1 \mathrm{~ms}$. The reason for the low contribution of the LS fibers to the total response is due to the relative contribution of the fiber types in the rate-level curve for moderate to high stimulus levels (see Fig.1(c)). 


\section{DISCUSSION}

The simulation results presented in this study confirm that at the single unit level, the latency-with-level function of the AN and IC responses reflects the underlying auditory filter bandwidth. These results are consistent with single-unit AN recordings that show decreased onset latencies for fibers recorded after noise-induced hearing loss than for normal fibers (Scheidt et al., 2010). However, this change in latency due to changes in auditory filter bandwidth was less pronounced at the level of the ABR wave-V, which only showed a $0.2 \mathrm{~ms}$ decrease in latency for moderate stimulus levels. This small effect is likely due to the overrepresentation of high-frequency channels that have short impulse responses, in the wide-band response. The ABR wave-V latency to broadband clicks may thus not be suitable for detecting hearing impairments related to wider-than-normal auditory filters. Because the latency of simulated single-unit responses did reflect changes in auditory filter bandwidth, ABRs to more frequencyspecific regions of the BM may be more powerful for diagnosing widening of peripheral auditory filters. This may explain why derived-band ABR wave-V latencies in response to 93-dB-peSPL clicks decreased with increasing pure-tone thresholds for high-pass noise cut-off frequencies of 0.7 and $1.4 \mathrm{kHz}$ (Don et al., 1998), whereas the NH and HI subject groups had similar wave-V latencies at high stimulus levels (Serpanos et al., 1997).

We found that LS fibers contribute little to the overall IC response. Even though response latencies of LS fibers were greater than the latencies of HS fibers (in agreement with findings of Rhode and Smith, 1985), their contribution to the total wave- $\mathrm{V}$ response was not substantial. The model employed a ratio of $50 \% \mathrm{HS}$ and LS fibers, with two rates, whereas it is probably more realistic to have a whole range of fibers with varying thresholds and rates represented (Liberman, 1978; Zilany et al., 2009). It is currently heavily debated what role LS fibers play in the perception of sound; especially as evidence suggesting that they become damaged by noise exposure before HS fibers (which are responsible for at-threshold responses) is growing (e.g., Furman, 2013). Unless the LS fibers make up a greater percentage of the total AN fiber population than is suggested by physiological estimates from cats $(61 \%$ HS and 34\% MS and LS; Liberman, 1978), or have saturation rates that exceed those for HS fibers at high stimulus levels (Liberman, 1978; Rhode and Smith, 1985), the model predicts that the LS fibers have a relatively modest contribution to the latency of both single-unit and wide-band IC responses. This also suggests that the latency of $\mathrm{ABR}$ wave- $\mathrm{V}$ will prove insensitive as a diagnostic tool for determining the status of the population of LS fibers in human patients.

\section{ACKNOWLEDGMENTS}

The authors would like to thank Mike Heinz and Filip Rønne for discussions related to this work. Financial support was received from the National Science and Engineering Faculty Fellowship (BGSC).

\section{REFERENCES}

Burkard, R. and Palmer, A.R. (1997). "Response of chopper units in the ventral cochlear nucleus of the anaesthetised guinea pig to click-in-noise and click trains," Hear. Res. 110, 234-250.

Dau, T. (2003). "The importance of cochlear processing for the formation of auditory brain-stem and frequency following responses," J. Acoust. Soc. Am. 113, 936-950.

Dolan D.F., Teas, D.C. and Walton, J.P. (1983). "Relation between discharges in auditory nerve fibers and the whole-nerve response shown by forward masking: An empirical model for the AP," J. Acoust. Soc. Am., 73(2), 580-591.

Don, M., Ponton, C. W., Eggermont, J. J., and Kwong, B. (1998). "The effects of sensory hearing loss on cochlear filter times estimated from auditory brainstem response latencies,” J. Acoust. Soc. Am. 104, 2280- 2289.

Donaldson, G. S., and Ruth, R. A. (1996). "Derived-band auditory brain- stem response estimates of traveling wave velocity in humans: II. Subjects with noise-induced hearing loss and Meniere's disease,” J. Speech Hear. Res. 39, 534-545.

Elberling, C., Callø, J., and Don, M. (2010). "Evaluating auditory brainstem responses to different chirp stimuli at three levels of stimulation," J. Acoust. Soc. Am. 128, 215-223.

Epp, B., Verhey, J. L., and Mauermann, M. (2010). "Modeling cochlear dynamics: Interrelation between cochlea mechanics and psychoacoustics,” J. Acoust. Soc. Am., 128(4), 1870-1883.

Furman, A.C. (2013). "Primary neuronal degeneration in the guinea pig: effects on spontaneous rate-type," PhD thesis, HarvardMIT.

Harte, J., Pigasse, G., and Dau, T. (2009). "Comparison of cochlear delay estimates using otoacoustic emissions and auditory brainstem responses,” J. Acoust. Soc. Am. 126, 1291-1301.

Heinz, M., Zhang, X., Bruce, I., and Carney, L. (2001). "Auditory nerve model for predicting performance limits of normal and impaired listeners," ARLO 5, 91-96. 
Henry, K.S., Kale, S., Scheidt, R.E., and Heinz, M.G. (2011). "Auditory brainstem responses predict auditory nerve fiber thresholds and frequency selectivity in hearing impaired chinchillas,"Hear.Res., 280, 236-244.

Ibrahim, R. A., and Bruce, I. C. (2010). "Effects of peripheral tuning on the auditory nerve's representation of speech envelope and temporal fine structure cues," in Neurophysiological Bases of Auditory Perception, edited by E. A. Lopez-Poveda and A. R. Palmer, 15th International Symposium on Hearing, Salamanca, Spain, 1-5 June, pp. 429-438, Med Elect. Hear Life.

Kujawa, S.G. and Liberman, M.C. (2009). "Adding insult to injury: cochlear nerve degeneration after "temporary" noise-induced hearing loss," J. Neurosc. 29, 14077-85.

Liberman, M.C. (1978), “Auditory-nerve response from cats raised in a low-noise chamber,” J. Acoust. Soc. Am. 63, $442-455$.

Moleti, A., Paternoster, N., Bertaccini, D., Sisto, R., and Sanjust, F. (2009). "Otoacoustic emissions in time-domain solutions of nonlinear non-local cochlear models," J. Acoust. Soc. Am. 126(5), 2425-2436.

Neely, S., Norton, S., Gorga, M., and, Jesteadt, W. (1988). "Latency of auditory brain-stem responses and otoacoustic emissions using tone-burst stimuli," J. Acoust. Soc. Am. 83, 652-656.

Nelson, P.C. and Carney, L.H. (2004). "A phenomenological model of peripheral and central neural responses to amplitudemodulated tones," J. Acoust. Soc. Am. 116(4), 2173-2186.

Recio, A., and Rhode, W. S. (2000). "Basilar membrane responses to broadband stimuli," J. Acoust. Soc. Am. 108(5), 22812298.

Rhode, W.S. and Smith, P.H. (1985), "Characteristics of tone-pip response patterns in relationship to spontaneous rate in cat auditory nerve fibers," Hear. Res. 18, 159-168.

Rønne, F.M., Dau, T., Harte, J.M., Elberling, C. (2012). "Modeling auditory evoked brainstem responses to transient stimuli," J. Acoust. Soc. Am. 131(5), 3903-3913.

Sachs, M.B. and Abbas, P.J. (1974). "Rate versus level functions for auditory nerve fibers in cats: Tone burst stimuli," J. Acoust. Soc. Am. 81, 680-691.

Serpanos, Y. C., O’Malley, H., and Gravel, J. S. (1997). "The relationship between loudness intensity functions and the clickABR wave V latency,” Ear Hear. 101, 2151-2163.

Scheidt, R.E., Kale S. and Heinz, M.G. (2010). "Noise-induced hearing loss alters the temporal dynamics of auditory-nerve responses," Hear. Res., 269, 23-33.

Strelcyk, O., Christoforidis, D. and Dau, T. (2009). "Relation between derived-band auditory brainstem response latencies and behavioral frequency selectivity," J. Acoust. Soc. Am. 124(4), 1878-1888.

van Hengel, P. W. J. (1996). "Emissions from cochlear modelling," PhD thesis, Rijksuniversiteit Groningen, Groningen.

Verhulst, S., Dau, T. and Shera, C.A. (2012). "Nonlinear time-domain cochlear model for transient stimulation and human otoacoustic emission," J. Acoust. Soc. Am., 132(6) 3842-3848.

Westerman, L. A., and Smith, R. L. (1988). "A diffusion model of the transient response of the cochlear inner hair cell synapse," J. Acoust. Soc. Am. 83, 2266-2276.

Zhang, X., Heinz, M.G., Bruce, I.C. and Carney, L.H. (2001). "A phenomenological model for the responses of auditory- nerve fibers: I. Nonlinear tuning with compression and suppression,” J. Acoust. Soc. Am. 109, 648-670.

Zilany, M.S.A., Bruce, I.C., Nelson, P.C., and Carney, L.H. (2009). "A Phenomenological model of the synapse between the inner hair cell and auditory nerve: Long-term adaptation with power-law dynamics," J. Acoust. Soc. Am. 126(5): $2390-2412$. 